\title{
Diseño y construcción de sistemas de predicción por conjuntos: error en las condiciones de contorno
}

DOI: $10.31978 / 014-18-009-X .18$

JUANMA SANCHO ÁviLA

Centro Nacional de Predicción (CNP), Agencia Estatal de Meteorología (AEMET)

En cualquier región delimitada los valores de contorno o frontera deberían especificarse para definir correctamente el problema, es decir, la solución debería depender de forma continua de las condiciones de contorno, de modo que pequeños cambios en los valores frontera produzcan a su vez pequeños cambios en la solución. Desafortunadamente [...] las ecuaciones diferenciales primitivas en que se basan usualmente los modelos atmosféricos son patológicas con respecto a las condiciones de contorno, debido a la aproximación hidrostática ...

Predicción numérica y meteorología dinámica - G. J. HALTINER Y R. T. WILliams

La predicción probabilista por conjuntos en alta resolución y a corto plazo es actualmente una herramienta clave para la predicción operativa en los servicios meteorológicos con responsabilidad en la emisión de avisos por tiempo severo. Aunque la capacidad computacional de los sistemas donde se ejecutan los modelos de predicción a escala global ha aumentado de forma muy importante en los últimos años y es previsible que la progresión continúe, la dependencia de las predicciones en alta resolución y a muy corto plazo en modelos de área limitada es muy probable que persista en el futuro. Por lo tanto, hoy por hoy, es una necesidad que los sistemas de predicción por conjuntos (SPC) basados en modelos de área limitada con resoluciones que se adentran en la mesoescala (modelos de malla kilométrica) se acoplen con SPC basados en modelos globales que proporcionen condiciones iniciales y de contorno adecuadas. Es importante, por lo tanto, realizar estudios con el fin de evaluar y conocer la influencia de la propagación de errores debido a la elección de condiciones de contorno en la calidad de las predicciones.

Palabras clave: diseño de sistemas de predicción por conjuntos, incertidumbres en las condiciones de contorno, archivo TIGGE. 


\subsection{Errores debidos a las condicio- nes de contorno}

En los modelos de área limitada existen importantes fuentes de error, ya tratadas en otras partes de este libro, que están basadas en el tratamiento de las aproximaciones en la dinámica del modelo, así como en las parametrizaciones de los procesos físicos o en las condiciones iniciales que incluyen tanto los errores en las observaciones como el sistema de asimilación de datos. Estas fuentes de error dependen exclusivamente de las estrategias diseñadas dentro del propio modelo de predicción. Sin embargo, los errores inducidos por las condiciones de contorno laterales (incluyendo las inferiores) son independientes de la sofisticación en el diseño del modelo de predicción encaminadas a limitar dichas fuentes de error, en otras palabras, son independientes del propio modelo, ya que dichas condiciones de contorno vienen dadas por otros modelos globales en los que se anida el modelo de área limitada. Existen numerosos estudios que insisten en que los errores propios de las condiciones de contorno proporcionadas por modelos globales se propagan dentro del dominio de los modelos de área limitada.

Entre los principales motivos de la inevitable propagación de errores por las condiciones de contorno laterales estarían los siguientes:

- La menor resolución espacial tanto horizontal como vertical de los modelos globales hace necesario interpolar espacial y temporalmente dichas condiciones a la resolución del modelo de área local con la consecuente degradación en la calidad de la información.

- En el dominio espectral, las mayores longitudes de onda propias de las condiciones de contorno laterales no interaccionan adecuadamente con las ondas de menor escala del modelo local, influyendo de forma negativa aumentando el error.

- Las mayores simplificaciones en el tratamiento de la física de los modelos globales así como las parametrizaciones utilizadas en ellos, que sí pueden estar ya resueltas en el diseño del modelo local, pueden provocar falsos gradientes ofeedbacks entre ambos grids que llevan necesariamente a una degradación de las predicciones dentro del dominio local.

Se estima que el error debido a las condiciones de contorno representa aproximadamente un $50 \%$ del error asociado al resto de fuentes de incertidumbre dentro de la primeras 24 h [20]. Sin embargo, la evaluación cuantitativa del peso relativo de estos errores depende fuertemente de las condiciones atmosféricas, de manera que por ejemplo, en condiciones de importantes forzamientos dinámicos de carácter local, los errores debidos a las condiciones de contorno se pueden reducir aunque continúen siendo considerables. Si las condiciones de contorno no se diseñan adecuadamente y en ciertas condiciones atmosféricas, los errores debidos a estas pueden afectar de tal manera a las predicciones dadas por el modelo de área local, que no mejoren las obtenidas por un modelo global como el del ECMWF a pesar de su menor resolución y las parametrizaciones menos sofisticadas utilizadas.

Por otra parte, el error debido a las condiciones de contorno crece de manera aproximadamente lineal con el tiempo hasta un punto en el que se satura y permanece uniforme. Este hecho es probablemente debido a que el error se va propagando internamente en el dominio hasta que finalmente todo es afectado manteniéndose constante a partir de entonces.

Aunque estas limitaciones son inevitables, sí se pueden afrontar distintos enfoques para el diseño de unas condiciones de contorno que minimicen en lo posible los errores propagados dentro del dominio local de predicción.

Es por lo tanto de importancia crítica diseñar y valorar las mejores estrategias basadas en dichos sistemas probabilistas globales que proporcionen suficiente dispersión para obtener condiciones laterales de contorno adecuadas en los sistemas probabilistas de área local a más alta resolución. El objetivo final es producir predicciones probabilistas que cuantifiquen suficientemente el grado de incertidumbre de las variables predichas [15].

A menudo los sistemas de predicción por conjuntos muestran un comportamiento subdispersivo, indicando que no cumplen el objetivo de reflejar con suficiente amplitud la diversidad de estados atmosféricos plausibles. Es decir, padecen de un déficit de spread o dispersión (sec. 15.8.2 en la página 223) en comparación con el error calculado para la muestra de los conjuntos de predicciones.

El diseño de unas adecuadas condiciones de contorno, es un factor más que afecta a la relación dispersiónerror siendo una buena elección de estas condiciones laterales la que proporcionara valores de dispersión comparables en magnitud con el error, como muestra de la existencia de consistencia estadística. 
En este estudio, se han probado distintas estrategias para la elección de adecuadas condiciones de contorno que se acerquen a este requisito y que se describirán a continuación.

Disponer de predicciones probabilistas de los Centros globales de predicción numérica del tiempo es imprescindible para realizar experimentos de distintas combinaciones y estrategias de diseño de condiciones de contorno apropiadas.

El Experimento de Investigación de los Sistemas de Observación y Predecibilidad THORPEX (Observing System Research and Predictability Experiment https://www . wmo. int/pages/prog/arep/ wwrp/new/thorpex_new.html), es un proyecto del Programa de Investigación del Tiempo Atmosférico Mundial (World Weather Research Programme) cuyo fin es mejorar la exactitud de las predicciones de eventos severos en alcances de 1 día a 2 semanas. En el marco de este proyecto se encuentra a su vez el proyecto TIGGE (THORPEX Interactive Grand Global Ensemble, ver cap. 26 en la página 387), consistente en el almacenamiento, gestión y distribución, para propósitos científicos, de datos de predicciones probabilistas por conjuntos procedentes de 10 centro globales de predicción numérica del tiempo (NWP). Dicho archivo contiene información desde octubre de 2006 hasta la actualidad y es gestionado y mantenido en el Centro Europeo de Predicción a Plazo Medio, ECMWF (European Centre for Medium-range Weather Forecasts, cap. 19 en la página 289). En este estudio se examinan diferentes experimentos basados en enfoques multimodelo utilizando datos de TIGGE y otras técnicas, como por ejemplo SLAF, Scaled Lagged Average Forecasting [6, 11, 17] que se describirán más adelante. Se lleva a cabo un estudio de la relación dispersión-error para predicciones con alcances de hasta 36 horas sobre la región de la península ibérica. La información utilizada se basa en datos tanto de análisis como de predicciones de varios parámetros correspondientes a los sistemas globales de predicción por conjuntos de Europa (ECMWF), Estados Unidos (NCEP), China (CMA), Canadá (CMC) y Reino unido (UKMO). Es decir, se han seleccionado 5 modelos globales de los 10 disponibles en TIGGE en base a la disponibilidad de datos para el periodo de estudio elegido. En este capítulo se describen los experimentos y se muestran los principales resultados. El resultado más importante y destacable de este estudio es que experimentos basados en conjuntos diseñados con predicciones procedentes de un único modelo global de los 5 seleccionados dan lugar a un comportamiento peor en términos de relaciones dispersión-error, y por lo tanto sufren de menor consistencia estadística. En otras palabras, las estrategias basadas en enfoques multimodelo se muestran más adecuadas para proporcionar condiciones laterales de contorno a un sistema de predicción por conjuntos de área local y alta resolución espacial. Otro resultado a destacar es la gran diferencia observada en el comportamiento de los diferentes modelos globales, aunque todos muestran como característica común, un comportamiento subdispersivo en que la dispersión es menor que el error (RMSE). Estos resultados animan a explorar diferentes experimentos multimodelo basados en técnicas LAF, Lagged Average Forecasting $[6,11,14]$. Esta técnica se basa en la utilización para un instante determinado de conjuntos de predicciones correspondientes a pasadas anteriores, con distintos alcances, para la estimación de las condiciones de contorno iniciales con el fin de ser introducidas en el sistema de predicción. Existe una variante de la anterior, la técnica SLAF (Scaled LAF, [6, 14, 17]), que tiene en cuenta la aplicación de factores de escala sobre las predicciones más antiguas para considerar la degradación de dichas predicciones. Estos factores de escala son menores al aumentar el alcance de las predicciones utilizadas. Los resultados preliminares indican que el uso de las técnicas SLAF suponen una mejora con respecto a las técnicas LAF. Para todos los parámetros estudiados la dispersión aumenta mientras que el error (RMSE, sec. 15.3 en la página 213) disminuye en el caso de los conjuntos formados por técnicas SLAF usando predicciones $\mathrm{T}+12$ junto con miembros de control $\mathrm{T}+0$. En este caso los valores de la dispersión son altamente dependientes de los factores de escala aplicados a los miembros de predicciones más antiguas, que como se dijo anteriormente se deben utilizar para tener en cuenta la degradación de las predicciones con el alcance. Por lo tanto, es crítica la elección de factores de escala adecuados en el diseño de los experimentos.

Existen estudios anteriores que merece la pena mencionar en los que ya se abordó el problema de la selección de condiciones de contorno adecuadas. A continuación se describen algunos de los resultados de estos enfoques.

Cuando se lleva a cabo la comparación de las características individuales de los modelos globales disponibles en el archivo TIGGE se observa una gran diferencia entre ellos. Los resultados preliminares apuntan a que el uso combinado de varios modelos para la creación se sistemas multimodelos por conjuntos puede 
mejorar cuantitativamente la calidad de las predicciones probabilistas mejorando de forma importante, por ejemplo, el comportamiento del modelo por conjuntos del ECMWF. Esto sucede especialmente en áreas y variables donde el ECMWF subestima la dispersión [16]. En el estudio de BuIZZA en 2005 [4] se realiza un estudio comparativo, para la primavera de 2002, del rendimiento y fiabilidad de los sistemas de predicción probabilistas basados en los modelos globales del ECMWF, MSC y NCEP, concluyendo que ninguno de ellos es capaz de capturar adecuadamente todas las fuentes de incertidumbre existentes en las predicciones, siendo la dispersión subestimada sistemáticamente cuando se consideran los tres modelos por separado. Otro trabajo más reciente, llevado a cabo por Hagedorn [10] llegó a la conclusión de que un sistema reducido multimodelo considerando miembros de solo 3 sistemas de predicción globales (USA, UK y ECMWF) supera en términos de calidad al sistema monomodelo del ECMWF pero, sin embargo, cuando se incluyen los nueve modelos de TIGGE en el diseño de dicho sistema multimodelo no se consigue una mejora con respecto al sistema del ECMWF.

Por otro lado GARCÍA-MOYA Y COL. [8], utilizando el Sistema multimodelo de la Agencia Estatal de Meteorología (AEMET) para corto plazo, diseñado con condiciones de contorno procedentes de diferentes sistemas de predicción, denominado AEMET Short Range Ensemble Prediction System, AEMET-SREPS [8], muestra de nuevo la mejora sustancial en términos de consistencia estadística con el incremento de la dispersión en un enfoque multimodelo con respecto a los comportamientos basados en un solo modelo. En el marco del desarrollo de sistemas de predicción por conjuntos en la mesoescala utilizando modelos de alta resolución de área limitada (LAM EPS), existen problemas adicionales relacionados con la insuficiente dispersión proporcionada, la cual es más evidente en el caso de fenómenos mesoescalares y en parámetros de alta variabilidad espacial y temporal como es el caso de la precipitación [7]. Un factor importante a tener en cuenta para explicar la falta de dispersión en dichos sistemas de área limitada es el uso de condiciones de contorno laterales no perturbadas (unperturbed LBC Lateral Boundary Conditions) procedentes de sistemas de predicción globales [12]. Mediante experimentos basados en el modelo perfecto y el análisis perfecto, con el fin de aislar y estudiar separadamente los efectos de los errores de la formulación del modelo y los debidos a los errores en las condicio- nes iniciales, se demostró [5] la importancia de las condiciones iniciales o de contorno para obtener una dispersión adecuada con el fin de mejorar la calidad del sistema de predicción probabilista. En el contexto del modelo COSMO se estudió [9] la evolución de la dispersión del sistema debido a perturbaciones en la formulación del modelo separadamente de las perturbaciones en las condiciones de contorno concluyendo que las condiciones de contorno comienzan a dominar la dispersión a partir de alcances de unas pocas horas, siendo la primeras horas más afectadas por las incertidumbres propias de la física del modelo. Es decir, que a medida que se avanza en el alcance de la predicción se hace más importante la consideración de las perturbaciones en las condiciones laterales de contorno. Por lo tanto, se hace evidente la necesidad de dedicar esfuerzos para un diseño adecuado de las condiciones de contorno laterales en sistemas de área limitada y alta resolución. Aquí se presentan resultados basados en técnicas Scaled Lagged Average Forecasting, SLAF [6] usando 5 modelos globales de TIGGE que muestran un incremento importante de la dispersión, que no está acompañado de un excesivo aumento del error, en contraste con otros métodos basados en la simple combinación de diferentes miembros procedentes de distintos modelos globales de TIGGE. Estos son resultados esperanzadores que apuntan a que este tipo de técnicas pueden ser muy apropiadas para abordar el problema de la falta de dispersión en sistemas probabilistas de área limitada y alta resolución.

\subsection{Archivo TIGGE y metodolo- gía}

Actualmente se archivan datos de 10 sistemas de predicción global en la base de datos de TIGGE. Todos estos sistemas de predicción por conjuntos están diseñados para simular el efecto de las principales fuentes de error en las predicciones numéricas del tiempo: las incertidumbres en las condiciones iniciales debidas a los errores observacionales y a las aproximaciones realizadas en el proceso de asimilación de datos para la obtención del análisis o estado inicial estimado de la atmósfera, y por otra parte, las incertidumbres del modelo propias de las aproximaciones en las ecuaciones que describen la física del mismo y las simplificaciones asociadas a las parametrizaciones de los procesos físicos en resoluciones mayores que el grid del modelo. Estos modelos son: ECMWF, NCEP, CMA, CMC, UKMO, BOM, CPTEC, JMA, KMA y Météo-France 
y cada uno de ellos esta basado en un número diferente de integraciones del modelo global. Una ejecución, llamada predicción de control, es alimentada inicialmente por un análisis no perturbado de la atmósfera durante el método de asimilación de datos, es decir que los datos observacionales son introducidos en el modelo sin ser modificados. El resto de integraciones, o miembros perturbados simulan, por una parte, los efectos de las incertidumbres en la medida de las observaciones y en algunos casos tienen también en cuenta las incertidumbres o errores propios de la física del modelo a través de la aplicación de perturbaciones estocásticas a las tendencias del modelo.

Este trabajo se ha llevado a cabo para un periodo de 6 meses utilizando predicciones diarias de junio a diciembre de 2011 y seleccionando 5 modelos del archivo TIGGE (ECMWF, NCEP, CMA, CMC y UKMO). El criterio de selección se ha basado en evitar periodos con falta de datos en el que todos los modelos dispongan de un periodo de predicciones común y al mismo tiempo se ha tratado de asegurar la mayor diversidad dinámica posible al tratarse de 5 sistemas de predicción globales diseñados usando un número diferente de miembros, así como enfoques distintos en la simulación de las fuentes de incertidumbre. En la Tabla 18.1 se muestran las principales características de los sistemas de predicción utilizados. En cuanto a los métodos de simulación de las incertidumbres en las condiciones iniciales el ECMWF utiliza un enfoque basado en vectores singulares [3]; el sistema de predicción chino, CMA, utiliza los llamados bred vectors o vectores criados [18]; el NCEP americano utiliza un método de transformación de ensembles con reescala, ETR [21]; el sistema inglés UKMO aplica un enfoque basado en filtros de KALMAN, Ensemble Transform Kalman Filter, ETKF [1], [19] y, finalmente, el sistema canadiense del CMC añade perturbaciones isotrópicas a las observaciones y genera análisis perturbados usando un filtro de KALMAN, Ensemble Kalman Filter, EnKF [13]. Todos los centros, salvo el CMA, tienen en cuenta el efecto de las incertidumbres del modelo mediante un enfoque de perturbaciones estocásticas [2] y el CMC aplica también diferentes parametrizaciones físicas (Dif. parametr. en la tabla) para los distintos miembros. En cuanto a la resolución espacial y el número de miembros, el ECMWF proporciona la mayor resolución horizontal $(0.28 \times 0.28$ grados) y vertical (62 niveles) además de exceder con creces el número de miembros (51) con relación al resto de los centros.

Se han llevado a cabo diferentes experimentos para el periodo de junio a diciembre de 2011 para la pasada de las $0 \mathrm{~h}$ y alcances de hasta $36 \mathrm{~h}$ cada $6 \mathrm{~h}$. Los modelos globales seleccionados de TIGGE son ECMWF, NCEP, CMA, CMC and UKMO y las variables evaluadas: mslp (presión en superficie), t $2 \mathrm{~m}$ (temperatura a 2 metros), sfcWind (viento a $10 \mathrm{~m}),(500,1000) \mathrm{gh}$ (alturas geopotenciales) y viento en $300 \mathrm{hPa}$ y $700 \mathrm{hPa}$. El dominio del experimento cubre la Región de la península ibérica (Figura 18.1) con una resolución espacial de $0.1^{\circ} \mathrm{x} 0.1^{\circ}$. El análisis del ECMWF ha sido utilizado como referencia en todos los casos para los cálculos del error (Root Mean Square Error, RMSE, sec. 15.3 en la página 213).

\begin{tabular}{lccccc}
\hline Centro & \#Miembros & Pert CCII & Pert modelo & Res hor / Res ver & \#Ejecuciones \\
\hline ECMWF (EU) & $1+50$ & SV+EDA & Física estocástica & TL639 $\left(0.28^{\circ} \times 0.28^{\circ}\right) / 62$ & $2(00 / 12)$ \\
NCEP (USA) & $1+20$ & ETR & Física estocástica & T190 $\left(0.90^{\circ} \times 0.90^{\circ}\right) / 28$ & $4(00 / 06 / 12 / 18)$ \\
CMA (China) & $1+14$ & BV & NO & T2 $2130\left(0.84^{\circ} \times 0.84^{\circ}\right) / 31$ & $2(00 / 12)$ \\
CMC (Canada) & $1+20$ & EnKF & Física estocástica + & $600 \times 300\left(0.6^{\circ} \times 0.6^{\circ}\right) / 40$ & $2(00 / 12)$ \\
& & & Dif. paramet. & \\
UKMO (UK) & $1+23$ & ETKF & Física estocástica & $\left(0.55^{\circ} \times 0.83^{\circ}\right) / 70$ & $2(00 / 12)$ \\
\hline
\end{tabular}

Tabla 18.1: Características del los modelos TIGGE (2016) para los 5 SPC utilizados. \#Miembros: Número de miembros perturbados ( 1 + indica un miembro adicional de control, no perturbado). Pert CCII: Método de perturbaciones de condiciones iniciales (ver texto). Pert modelo: Método de simulación del error del modelo (ver texto). Res hor: Resolución horizontal. Res ver: Resolución vertical, medida en número de niveles verticales del modelo. 
Campo espacial. Denotamos un campo espacial por $\phi_{m}^{(n)}(x, t)$, donde $\phi$ es la variable, $n$ es el número de predicciones (en este caso una por día desde el 1 de junio al 31 de diciembre), $m$ es el número de miembros o predicciones en el experimento definido y t es el alcance $(0,6,12,18,24,30$ ó $36 \mathrm{~h})$. Estamos interesados en valorar el comportamiento de la dispersión del conjunto de predicciones con respecto a la media de todas la predicciones como medida de la incertidumbre del sistema de predicción por conjuntos, y su relación con el error medio de las predicciones comparadas con el análisis del ECMWF. Podemos tomar esta relación dispersión-error como una medida indirecta de la predecibilidad de la atmósfera. Con este propósito, cada campo $\phi_{m}^{(n)}(x, t)$ ha sido discretizado espacialmente $\phi_{i, m}^{(n)}(t)=\phi\left(x_{i, m}^{(n)}, t\right)$, siendo $i=1,2, \ldots, L$, donde $L$ es el número de puntos de grid, definido en el dominio de la península ibérica de límites: $35 \mathrm{~N} / 44 \mathrm{~N} / 11 \mathrm{~W} / 5 \mathrm{E}$ con una resolución espacial de $0,1^{\circ} \times 0,1^{\circ}$.

Dispersión. Usamos la desviación estándar como medida de dispersión (spread):

$\operatorname{stddev}(t)=\left(\frac{1}{N} \sum_{n=1}^{N}\left(\frac{1}{m-1} \sum_{m=1}^{M}\left(\phi_{i, m}^{(n)}(t)-\overline{\phi_{i}^{(n)}(t)}\right)^{2}\right)\right)^{\frac{1}{2}}$

donde $N$ es el número total de predicciones (en nuestro caso, días de predicción en el periodo), $M$ es el número total de miembros del SPC diseñado y $\overline{\phi_{i}^{(n)}(t)}$ es la media del ensemble o conjunto de todas las predicciones proporcionadas por los distintos miembros para un mismo alcance:

$$
\overline{\phi_{i}^{(n)}(t)}=\frac{1}{M} \sum_{m=1}^{M} \phi_{i, m}^{(n)}(t)
$$

Error. Como medida de error usamos la raíz cuadrada del error cuadrático medio (Root Mean Square Error, RMSE, sec. 15.3 en la página 213) del siguiente modo:

$$
\operatorname{RMSE}(t, i)=\sqrt{\frac{1}{N} \sum_{n=1}^{N}\left(\overline{\phi_{i}^{(n)}(t)}-\phi_{i, a n}^{(n)}(t)\right)^{2}}
$$

donde $\phi_{i, a n}^{(n)}(t)$ es el análisis de la variable $\phi$ obtenido por el ECMWF correspondiente a la predicción $n^{\text {th }}$, al punto de grid $i$ y a al alcance $t$.

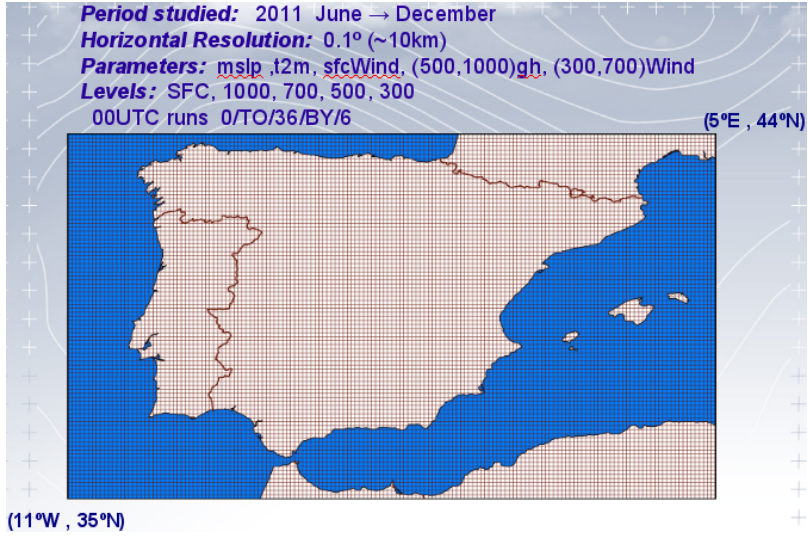

Figura 18.1: Dominio, resolución espacial, variables y niveles utilizados.

El objetivo principal del estudio es comparar diferentes combinaciones de conjuntos de predicciones generadas por diferentes centros siguiendo enfoques tanto monomodales como multimodales utilizando técnicas LAF y SLAF y valorar para cada caso, el comportamiento de la relación dispersión-error como medida de la consistencia estadística de cada diseño o experimento.

\subsection{Comparación de los diferen- tes sistemas de predicción por conjuntos en TIGGE}

Como primer paso y con el fin de tener una primera impresión acerca del comportamiento de las curvas dispersión-error para cada uno de los sistemas de predicción en cada centro de forma individual, se han construidos conjuntos monomodelos. Para ello se han seleccionado aleatoriamente 10 miembros perturbados para cada de uno de los 5 modelos globales TIGGE elegidos. Se han utilizado el mismo número de miembros (10) para tener conjuntos con la misma dimensión. Los modelos seleccionados fueron: ECMWF, NCEP, CMA, CMC y UKMO mostrándose las curvas dispersión-error para la presión al nivel del mar y las componentes u y v de la velocidad del viento a $10 \mathrm{~m}$ en la Figura 18.2. En resumen, se observa un mejor comportamiento del sistema del ECMWF en comparación con el resto de centros mundiales seleccionados, mostrando todos ellos características subdispersivas. El sistema del ECMWF es menos subdispersivo que el resto, mostrando una más clara relación lineal entre la dispersión y el error, lo cual indica una mayor consistencia estadística con las observaciones (análisis). 
Sin embargo, se puede obtener un mejor comportamiento formando un conjunto multimodelo construido con los 5 miembros de control de cada uno de los modelos, excluyendo cualquier miembro perturbado. Este sistema, aun teniendo la mitad de miembros, mejora cualquier diseño basado en un solo modelo, incluso al propio ECMWF, mostrando un clara mejora en cuanto a la fiabilidad del sistema frente a cualquier diseño monomodelo con valores mayores de dispersión para los distintos alcances sin aumentar el error (RMSE). Por otro lado, este sistema de 5 miembros de control muestra un destacable comportamiento ligeramente sobredispersivo para, por ejemplo, la altura geopotencial en $1000 \mathrm{hPa}$ y $500 \mathrm{hPa}$ (ver línea negra en la Figura 18.3).

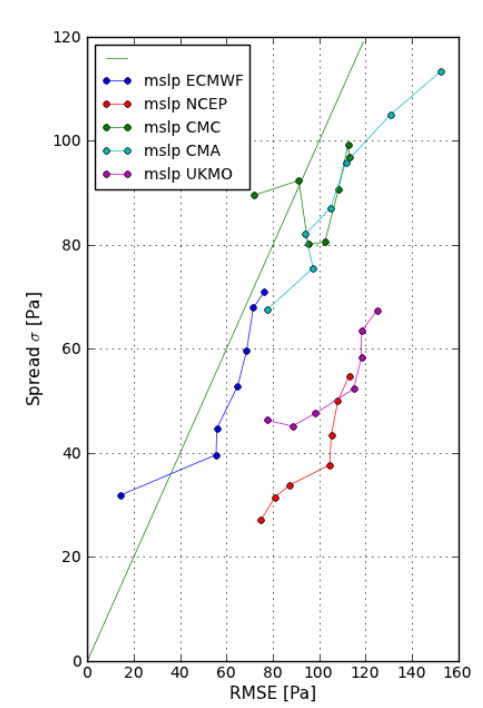

SPREAD-SKILL msIp,10u,10v [Jun->Dic 2011]
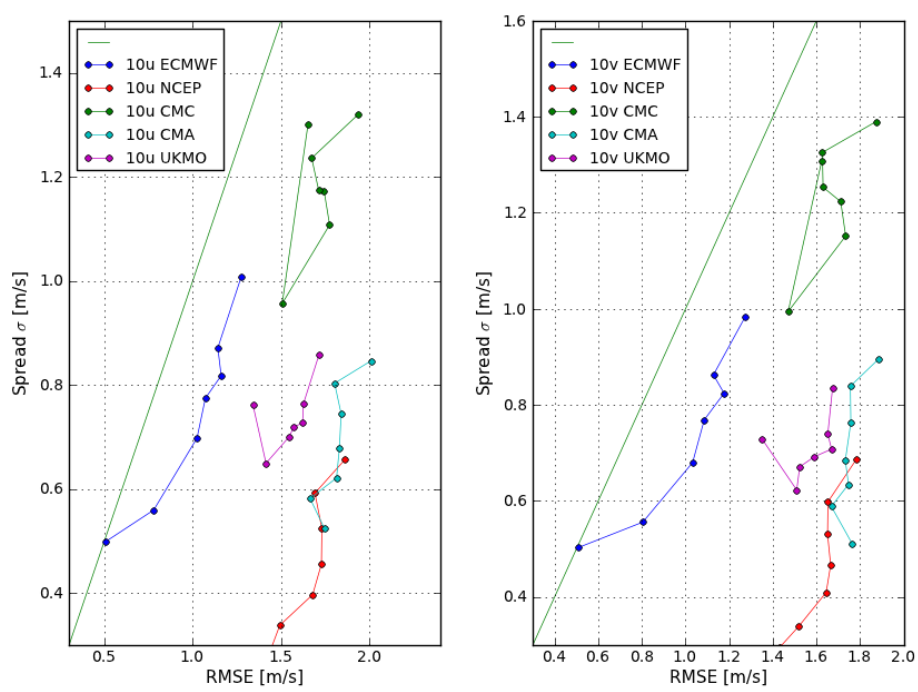

Figura 18.2: Curvas dispersión-error para la presión a nivel del mar (mslp), componente zonal del viento (10u) y latitudinal (10v). Los conjuntos están formados por 10 miembros perturbados elegidos aleatoriamente para cada uno de los 5 sistema(s) de predicción por conjuntos (SPC) globales TIGGE.
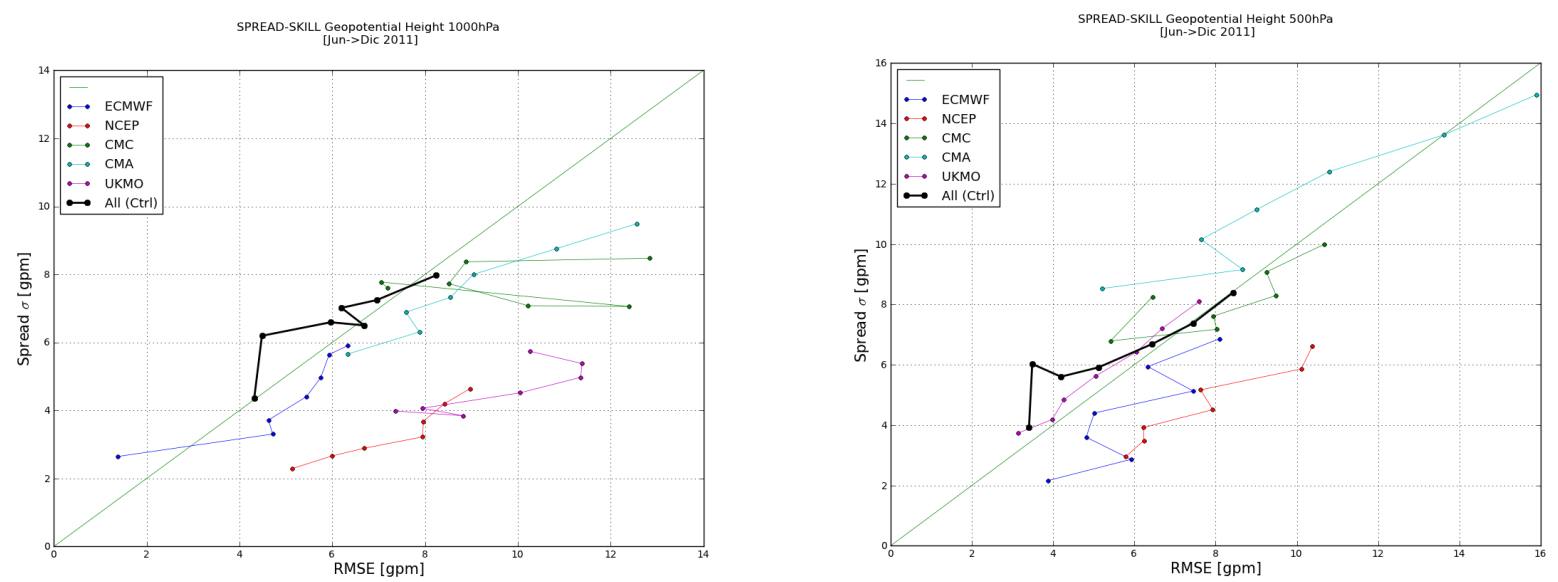

Figura 18.3: Relaciones dispersión-error para la altura geopotencial en 1000 hPa y 500 hPa. En colores se representan los conjuntos formados usando 10 miembros aleatorios para cada uno de los 5 SPC globales TIGGE. La linea negra muestra el dispersión-error para un conjunto multimodelo formado usando únicamente los 5 miembros de control de cada modelo (excluyendo los miembros perturbados) 


\begin{tabular}{|c|c|c|}
\hline Experimento & Descripción del diseño & \# Miembros \\
\hline Tigge_5 & 5 miembros de ctrl $(\mathrm{T}+00)$, uno por cada modelo & $5 \mathrm{ctrl}$ \\
\hline Tigge_15 & $\begin{array}{c}5 \text { miembros de ctrl }(\mathrm{T}+00) \& 2 \text { miembros pert. }(\mathrm{T}+00) \\
\text { para cada modelo }\end{array}$ & $5 \mathrm{ctrl}+2 \times 5$ pert. $=15$ \\
\hline TiggeSlaf_10 & $\begin{array}{l}5 \text { miembros de ctrl }(\mathrm{T}+00) \& 5 \text { predicciones de ctrl. } \\
(\mathrm{T}+12) \mathrm{LAF}\end{array}$ & $5 \mathrm{ctrl}+5 \mathrm{fc}(\mathrm{T}+12)=10$ \\
\hline TiggeSlaf_15 & $\begin{array}{l}5 \text { miembros de } \operatorname{ctrl}(\mathrm{T}+00) \& 10 \text { predicciones de ctrl. } \\
(\mathrm{T}+12, \mathrm{~T}+24) \mathrm{LAF}\end{array}$ & $\begin{array}{c}5 \mathrm{ctrl}+5 \mathrm{fc}(\mathrm{T}+12)+ \\
5 \mathrm{fc}(\mathrm{T}+24)=15\end{array}$ \\
\hline TiggeFc_5 & 5 predicciones de ctrl. $(\mathrm{T}+06) \mathrm{LAF}$ & $5 \mathrm{fc}(\mathrm{T}+06)$ \\
\hline TiggeFc_10 & 10 predicciones de ctrl. $(\mathrm{T}+06, \mathrm{~T}+18) \mathrm{LAF}$ & $5 \mathrm{fc}(\mathrm{T}+06)+5 \mathrm{fc}(\mathrm{T}+18)=10$ \\
\hline
\end{tabular}

Tabla 18.2: Diseño de los experimentos LAF-

\subsection{Experimentos multimodelo LAF y SLAF}

Los buenos resultados obtenidos cuando se consideran enfoques multimodelo animan a recomendar explorar otras combinaciones para el diseño de conjuntos de predicciones usando miembros de los 5 modelos TIGGE. Se han diseñado nuevos conjuntos para comparar las relaciones dispersión-error (sec. 15.8.2 en la página 223) usando solo miembros no perturbados, o de control, miembros de control añadiendo algunos miembros perturbados así como métodos Lagged Average Forecasting (LAF) basados en el uso de predicciones pasadas [11]. También se han realizado experimentos combinados SLAF [6] junto con miembros de control. Ver Tabla 18.2 para la descripción de los experimentos.

El experimento TIGGE_5 consiste en un conjunto de 5 miembros formados por los miembros de control de cada modelo (análisis de baja resolución con alcance $0 \mathrm{~h}$ ) para los 5 modelos globales y pasadas de las 00 y 12 UTC. Hubiera sido deseable utilizar campos de análisis de alta resolución cada 6 h para este experimento pero en el archivo Meteorological Archival and Retrieval System (MARS) del ECMWF solo están disponibles los miembros de control cada $12 \mathrm{~h}$. El segundo experimento, llamado TIGGE_15 está formado añadiendo al conjunto anterior 2 miembros perturbados (alcance $0 \mathrm{~h}$ ) por cada modelo global formando un conjunto de 15 miembros. El experimento llamado TIGGESLAF_10 es el resultado de añadir 5 predicciones de los controles de la pasada de las $12 \mathrm{~h}$ anteriores al conjunto del experimento TIGGE5 obteniendo un conjunto LAF de 10 miembros. Es importante destacar en este punto que este experimento no es un conjunto SLAF propiamente dicho, ya que no se aplican factores de escala dependientes de la antigüedad de las predicciones para dar cuenta la degradación de la cali- dad de las predicciones con el alcance de las mismas. El experimento TIGGESLAF_15 simplemente añade miembros de predicciones $\mathrm{h}+24$ al anterior, pero tampoco es un experimento SLAF propiamente dicho ya que tampoco aplica factores de escala decrecientes a los miembros de predicciones para evitar que el conjunto este contaminado por errores del predicciones antiguas. Finalmente, los experimentos TIGGE_FC_5 y TIGGE_FC_10 están formados con 5 predicciones de control $\mathrm{h}+6$ en el primer caso y añadiendo a las anteriores 5 predicciones de control $\mathrm{h}+18$ en el segundo. En la segunda parte de este estudio se emplea la técnica SLAF construyendo conjuntos añadiendo miembros simétricamente alrededor de los miembros de control y escalados apropiadamente dependiendo del alcance de las predicciones para tener en cuenta la degradación de las predicciones.

Las relaciones dispersión-error para el geopotencial en $500 \mathrm{hPa}$ para cada uno de los experimentos descritos son mostrados en la Figura 18.4 en la página siguiente. Cada punto, de diferente color para cada experimento, es el resultado del cálculo de la desviación estándar con respecto a la media como medida de la dispersión, y el error cuadrático medio (Root Mean Square Error) de la media de los ensembles con respecto al análisis del centro europeo es usado como referencia para las pasadas de las 00 y $12 \mathrm{~h}$. Se estima que el número de valores utilizados para la computación de cada uno de los puntos en la gráfica es aproximadamente de 16000000 valores (14 651 puntos de grid x 182 días x 5 modelos + análisis del ECMWF (14 $651 \times 182)$ ). La linea negra representa la evolución del dispersión-error para un alcance máximo de $36 \mathrm{~h}$ y un paso de tiempo de $6 \mathrm{~h}$ para el caso de los 5 miembros de control de la pasada de las $0 \mathrm{~h}$ para cada uno de los modelos en un enfoque multimodelo. El primer y segundo punto de a linea negra obviamente coincide con los puntos $\mathrm{T}=0 \mathrm{~h}$ y $\mathrm{T}=6 \mathrm{~h}$ de los experimentos TIGGE_5 y TIGGEFC_5 respecti- 
vamente. Las diferencias observadas en el RMSE son debidas a errores sistemáticos o bias de los miembros de control $(\mathrm{T}+00)$ de los modelos globales con respecto a los valores de los análisis del ECMWF. Por ejemplo, en el caso de la temperatura a $2 \mathrm{~m}$ la mayoría de los modelos globales excepto NCEP y UKMO muestran sistemáticamente a las 12 UTC temperaturas inferiores que el análisis del ECMWF, en amarillo, mientras que por el contrario, la distribución en el caso de la pasada de las 00 UTC es aproximadamente simétrica alrededor el análisis del ECMWF (ver izquierda de Figura 18.5). Sin embargo, las diferencias en la dispersión no son importantes entre las 00 UTC y las 12 UTC (ver derecha de Figura 18.5). Estas diferencias también han sido observadas en el caso de otras variables.

Para el caso de la altura geopotencial en $500 \mathrm{hPa}$, el experimento que muestra los valores más bajos de dispersión y error es TIGGE_5, formado por 5 miembros de control de los modelos globales para alcance $0 \mathrm{~h}$. Se observa un importante aumento de la dispersión sin efectos claros en el aumento del error en el caso del experimento TIGGE_15, el cual añade 2 miembros perturbados para cada modelo global. Los experimentos LAF muestran errores algo mayores y dispersiones intermedias. Los resultados son mucho más esperan- zadores, en cuanto a la mejora de la consistencia estadística, cuando en la segunda parte de este trabajo los experimentos LAF se convierten a SLAFS con el resultado de una disminución sustancial del error. Los experimentos que incluyen solo miembros predictivos (TIGGEFC_5 y TIGGEFC_10) son los que tienen asociados los valores mayores de la dispersión y TIGGEFC_10 muestra mayores valores de RMSE probablemente por el efecto de la degradación debida a añadir predicciones mas antiguas, $\mathrm{T}+18$.

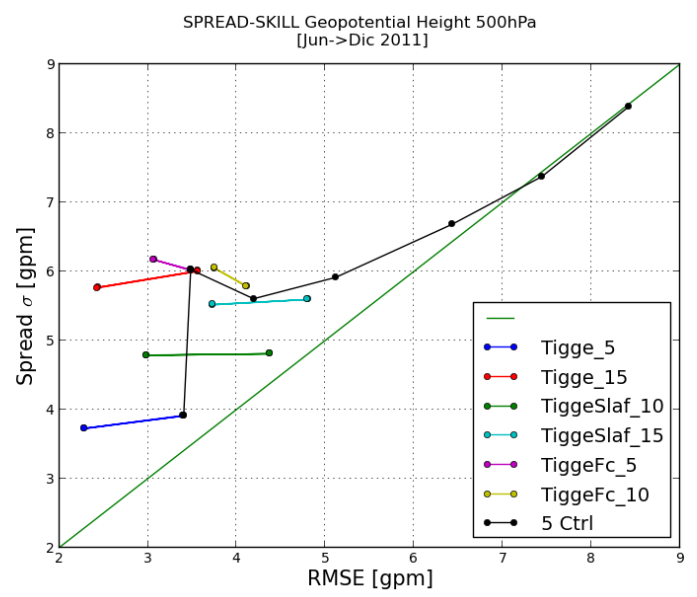

Figura 18.4: Relaciones dispersión-error para experimentos LAF.
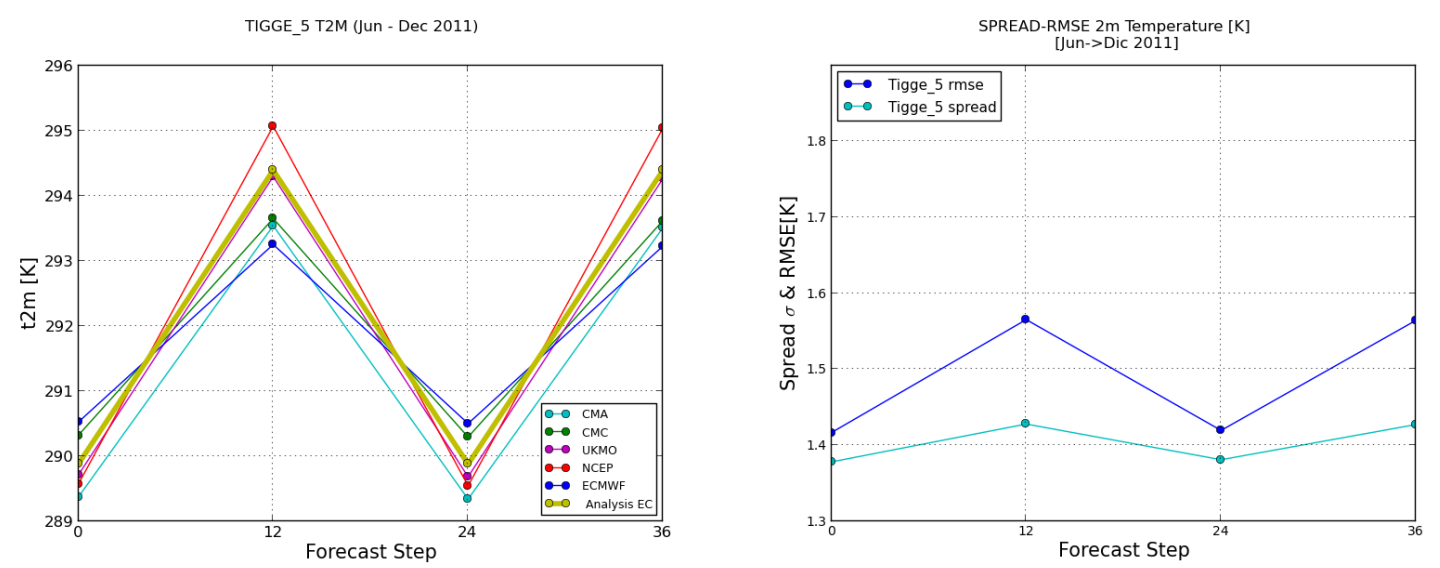

Figura 18.5: Izquierda: T2m de los modelos globales vs. análisis del ECMWF, derecha: TIGGE_5 t2m dispersiónerror

\begin{tabular}{|c|c|c|c|c|}
\hline LAF & SLAF & $K_{12}, K_{24}$ & Descripción del experimento & \# Miembros \\
\hline \multirow{2}{*}{$\begin{array}{l}\text { TiggeSlaf_10 } \\
(\text { solo } \mathrm{T}+12)\end{array}$} & TiggeSlaf_10R & $K_{12}=0,75$ & \multirow{2}{*}{$5 \operatorname{ctrl}[00] \& 5 \times\left\{c t r l[00] \pm K_{12}(\operatorname{ctrl}[00]-f c[12])\right\}$} & \multirow{2}{*}{15} \\
\hline & TiggeSlaf_10R2 & $K_{12}=1$ & & \\
\hline \multirow{2}{*}{$\begin{array}{l}\text { TiggeSlaf_15 } \\
(\mathrm{T}+12 \text { y } \mathrm{T}+24)\end{array}$} & TiggeSlaf_15R & $K_{12,24}=0,75,0,25$ & \multirow{2}{*}{$\begin{array}{c}5 \operatorname{ctrl}[00] \& 5 \times\left\{\operatorname{ctrl}[00] \pm K_{12}(\operatorname{ctrl}[00]-f c[12])\right\} \& \\
5 \times\left\{\operatorname{ctrl}[00] \pm K_{24}(\operatorname{ctrl}[00]-f c[24])\right\}\end{array}$} & \multirow{2}{*}{25} \\
\hline & TiggeSlaf15R2 & $K_{12,24}=1,0,5$ & & \\
\hline
\end{tabular}

Tabla 18.3: Diseño de experimentos multimodelo mediante la técnica SLAF 
Como se describió anteriormente, los experimentos TIGGESLAF_10 y TIGGESLAF_15 son el resultado de añadir a TIGGE_5 nuevos miembros con predicciones de control $([\mathrm{T}+12]$ y $[\mathrm{T}+12, \mathrm{~T}+24]$ respectivamente) formando siguiendo la técnica LAF (Lagged Average Forecasting). Para tener en cuenta la degradación en la precisión de las predicciones según el alcance de la predicción se deberían aplicar unos factores de escala que disminuyan el peso de dichas predicciones con la antigüedad de las predicciones en el conjunto de los miembros del sistema. Estos factores serían dependientes del alcance, disminuyendo para predicciones más antiguas. Esta técnica, que es por lo tanto, una variante mejorada de la técnica LAF denominada SLAF (Scaled Lagged Average Forecasting). Con el fin de explorar los posibles beneficios de la aplicación de esta técnica en la mejora de la consistencia estadística se han diseñado 4 nuevos experimentos SLAF denominados TIGGESLAF_10R, TIGGESLAF_15R, TIGGESLAF10R2 y TIGGESLAF15R2. El cuadro 18.3 muestra la descripción de dichos experimentos en los que la idea principal es la aplicación de factores de escala $K$ en el rango 0-1 a los miembros que proceden de predicciones.

Además, se amplía el número de miembros procedentes de predicciones de forma simétrica con respecto al análisis (control $\mathrm{T}+0$ ) sumándole y restándole perturbaciones de predicciones anteriores, aplicándoles factores de escala dependientes del alcance para evitar que el conjunto este excesivamente contaminado por predicciones antiguas. Con este fin, se han probado 2 series de factores de escala (ver Tabla 18.3). Los factores $K_{12}=0,75$ y $K_{24}=0,25$ utilizados en los experimentos $10 \mathrm{R}$ y $15 \mathrm{R}$, son los usuales y más frecuentemente utilizados en este tipo de sistemas de predicción por conjuntos mientras que los valores más altos $K_{12}=1$ y $K_{24}=0,5$ son los utilizados en el experimento SAMEX 98 [12]. Como consecuencia de añadir miembros simétricos con respecto al análisis, se incrementa significativamente el número de miembros del ensemble en este tipo de experimentos, de manera que se obtienen 15 miembros en el caso de TIGGESLAF10R y 25 miembros en el experimento TIGGESLAF15R.

Hay algunos efectos destacables y muy positivos en las relaciones dispersión-error como consecuencia del cambio de la técnica LAF a SLAF en el diseño de los experimentos. Para todas las variables estudiadas (Z500, mslp, t2m, velocidad del viento [sfc,700]) la dispersión (desviación estándar) aumenta mientras que el error (RMSE) disminuye de forma sensible para el caso de los experimentos SLAF usando predicciones $\mathrm{T}+12$ junto con miembros de análisis o control (experimentos TIGGESLAF10R y TIGGESLAF10R2). Obviamente, este incremento de la dispersión es mucho más acusado cuando los factores de escala elegidos son mayores (experimento TIGGESLAF10R2), pero sin embargo los valores del error no se ven afectados en absoluto, probablemente debido a que los miembros están simétricamente distribuidos alrededor de los miembros de control. Estos cambios positivos en el comportamiento de la dispersión y el error son más evidentes en el caso del viento en superficie, en $700 \mathrm{hPa}$ y en la temperatura a $2 \mathrm{~m}$. Un comportamiento sobredispersivo en los diagramas dispersión-error y los valores más altos de la dispersión se observan en estos experimentos (Figuras 18.6, 18.7 y 18.8). En el caso de la altura geopotencial en $500 \mathrm{hPa}$ y presión a nivel del mar (Figuras 18.9 y 18.10) también se observan valores inferiores de dispersión y error aunque los cambios no son tan evidentes para estas variables y la dispersión todavía es inferior a la obtenida en el experimento TIGGE_15 (que contenía miembros perturbados) con un error similar. Los experimentos TIGGEFC_5 y TIGGEFC_10 con técnicas LAF, con miembros de predicción, proporcionan altos niveles de dispersión pero incrementando los errores al mismo tiempo. Con respecto a los experimentos SLAF que incluyen predicciones $\mathrm{T}+24$, se produce una disminución del error en comparación con los experimentos LAF pero la dispersión disminuye a pesar del aumento en el número de miembros, posiblemente debido a los valores de escala más bajos aplicados a las predicciones más antiguas a $24 \mathrm{~h}$.

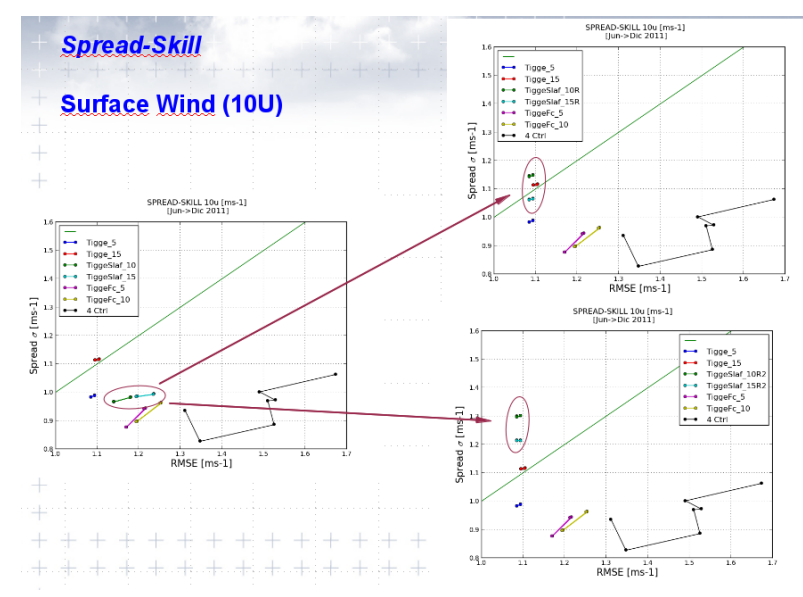

Figura 18.6: Relaciones dispersión-error para la componente u del viento a 10 metros. Los experimentos $L A F$ a la izquierda y SLAF a la derecha usando diferentes factores de escala. 


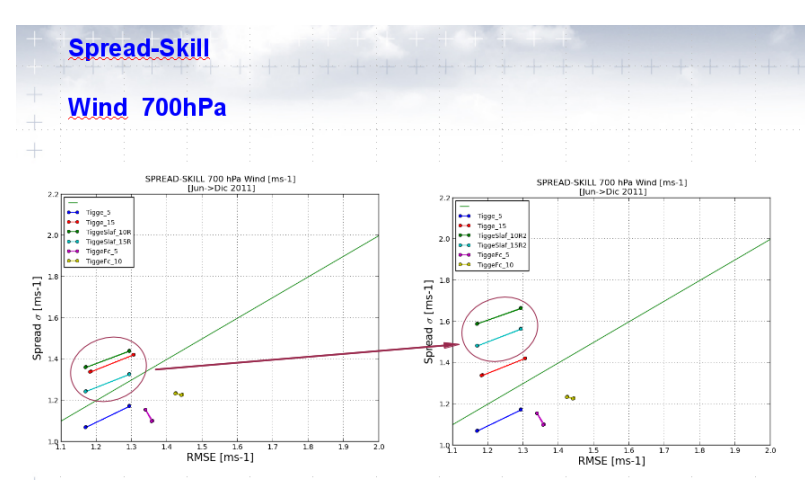

Figura 18.7: Relaciones dispersión-error para el viento en $700 \mathrm{hPa}$. Los experimentos LAF a la izquierda y SLAF a la derecha usando diferentes factores de escala.

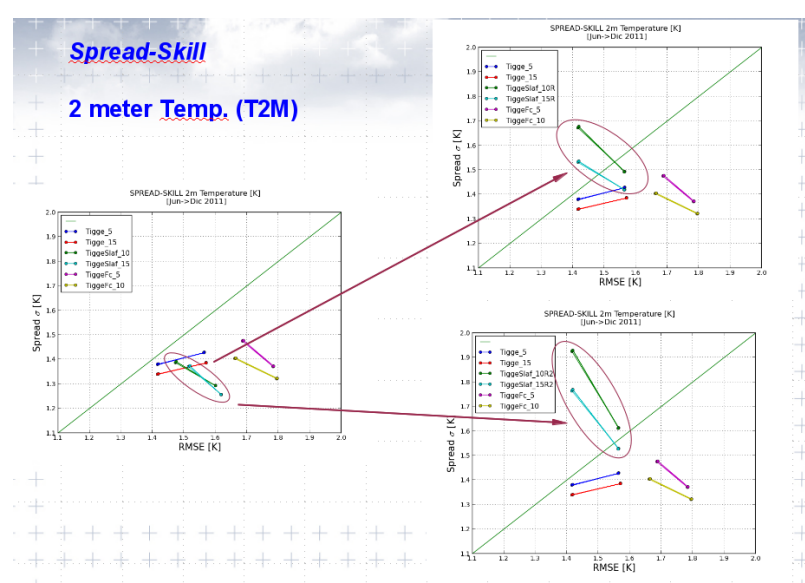

Figura 18.8: Relaciones dispersión-error para la temperatura a 2 metros. Los experimentos LAF a la izquierda y SLAF a la derecha usando diferentes factores de escala.

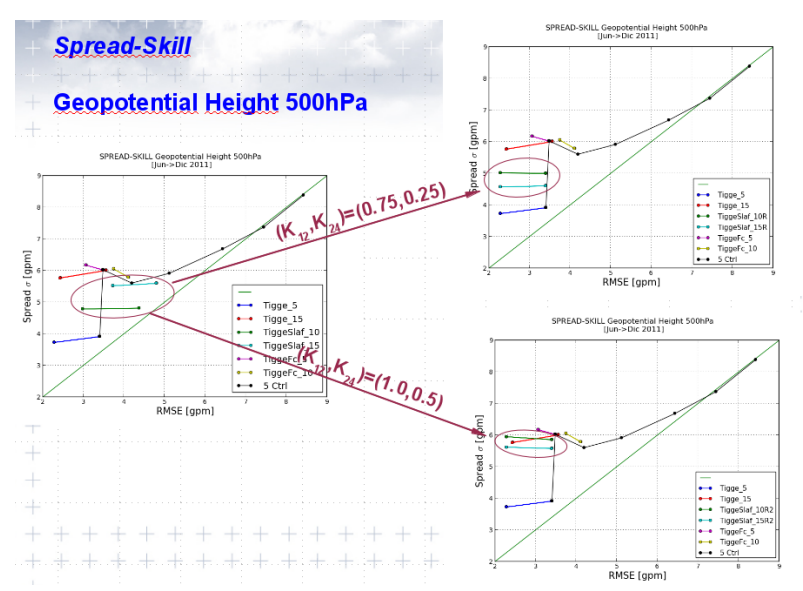

Figura 18.9: Relaciones dispersión-error para la altura geopotencial en $500 \mathrm{hPa}$. Los experimentos LAF a la izquierda y SLAF a la derecha usando diferentes factores de escala.

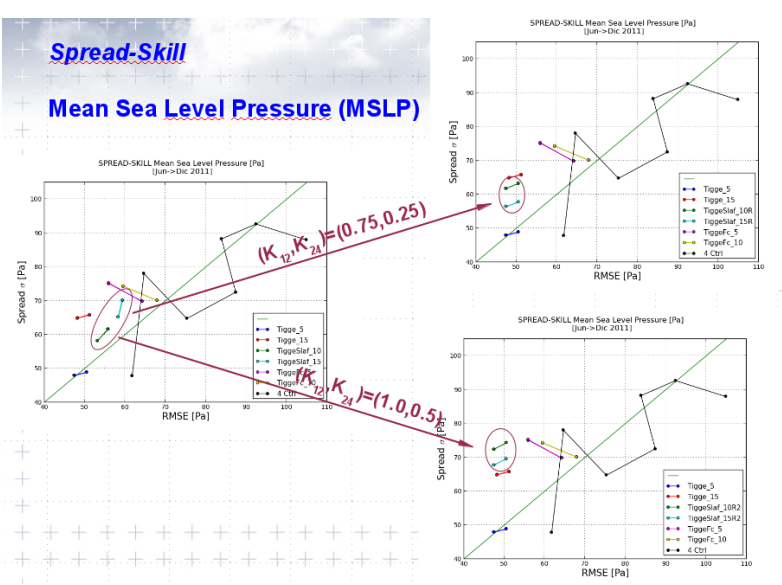

Figura 18.10: Relaciones dispersión-error para la presión a nivel del mar. Los experimentos LAF a la izquierda y SLAF a la derecha usando diferentes factores de escala.

\subsection{Conclusiones}

El ECEPS (sec. 19.3 en la página 293) se comporta mejor en términos de consistencia estadística que el resto de sistemas globales de predicción por conjuntos: CMA, CMC, UKMO y NCEP archivados en TIGGE (cap. 26 en la página 387). En todos los casos se observa un comportamiento subdispersivo en cuanto a la relación dispersión-error, siendo siempre la dispersión menor que el error. Sin embargo, un enfoque multimodelo formando un conjunto de miembros usando solo los miembros de control de cada uno de los 5 modelos globales seleccionados, se comporta mejor que el SPC del ECMWF (y que todos los demás), aún siendo ligeramente sobredispersivo. En la segunda parte del trabajo se profundiza en el enfoque multimodelo con nuevos experimentos diseñados combinando miembros de control para $\mathrm{T}+0, \mathrm{~T}+12$ y $\mathrm{T}+24$ con miembros perturbados $(\mathrm{T}+0)$. Se utiliza también la técnica SLAF (Scaled Lagged Average Forecasting), que proporcionó valores altos de dispersión (medida con la desviación estándar) junto con bajos errores (medidos con RMSE). El único experimento que utiliza miembros perturbados junto con miembros de control (TIGGE_15) proporciona resultados demasiado sobredispersivos. Obviamente, los factores de escala usados para disminuir la influencia del error según el alcance de las predicciones a $\mathrm{T}+12$ y $\mathrm{T}+24$, afectan de manera importante a la dispersión (pero no al error, probablemente debido a la aplicación de forma simétrica de perturbaciones alrededor de los miembros de control), lo cual indica que se debe prestar especial cuidado en la elección de los valores de los factores de escala con el fin no degradar los conjuntos. 


\subsection{Referencias}

[1] Bishop, Craig H., Etherton, Brian J y MAJUmDAR, Sharanya J. "Adaptive sampling with the ensemble transform Kalman filter. Part I: Theoretical aspects". En: Monthly weather review 129.3 (2001), páginas 420-436 (citado en página 279).

[2] Buizza, Roberto, Miller, Martin J y PalMER, Tim N. "Stochastic representation of model uncertainties in the ECMWF ensemble prediction system". En: Quarterly Journal of the Royal Meteorological Society 125.560 (ago. de 1999), páginas 2887-2908. ISSN: 00359009. DOI: 10 . 1002 / qj . 49712556006 (citado en página 279).

[3] Buizza, Roberto y Palmer, Tim N. "The Singular-Vector Structure of the Atmospheric Global Circulation". En: Journal of the Atmospheric Sciences 52.9 (mayo de 1995), páginas 1434-1456. ISSN: 0022-4928. DOI: 10 . 1175/1520-0469(1995) 052<1434: TSVSOT>2 . 0 . CO; 2 (citado en página 279).

[4] BuizzA, Roberto y col. "A Comparison of the ECMWF, MSC, and NCEP Global Ensemble Prediction Systems". En: Monthly Weather Review 133.5 (mayo de 2005), páginas 1076-1097. ISSN: 0027-0644. DOI: 10. 1175/MWR2905. 1 (citado en página 278).

[5] Clark, Adam J. y col. "Contributions of Mixed Physics versus Perturbed Initial/Lateral Boundary Conditions to Ensemble-Based Precipitation Forecast Skill". En: Monthly Weather Review 136.6 (jun. de 2008), páginas 2140-2156. ISSN: 0027-0644. DOI: 10 .1175/2007MWR2029 . 1 (citado en página 278).

[6] Ebisuzaki, W y Kalnay, Eugenia. "Ensemble experiments with a new lagged average forecasting scheme". En: WMO Research Activities in Atmospheric and Oceanic Modeling Rep 15 (1991), página 308 (citado en páginas 277, 278, 282).

[7] ECKel, F Anthony y Mass, Clifford F. "Aspects of effective mesoscale, short-range ensemble forecasting". En: Weather and Fore- casting 20.3 (2005), páginas 328-350 (citado en página 278).

[8] GarcíA-Moya, José Antonio y col. "Predictability of short-range forecasting: A multimodel approach". En: Tellus, Series A: Dynamic Meteorology and Oceanography 63.3 (mayo de 2011), páginas 550-563. ISSN: 02806495. DOI: $10.1111 / \mathrm{j} .1600-0870$. 2010.00506.x (citado en página 278).

[9] Gebhardt, C. y col. "Uncertainties in COSMO-DE precipitation forecasts introduced by model perturbations and variation of lateral boundaries". En: Atmospheric Research 100.2-3 (mayo de 2011), páginas 168-177. ISSN: 0169-8095. DOI: 10 . 1016/J . ATMOSRES . 2010 . 12.008 (citado en página 278).

[10] Hagedorn, Renate y col. "Comparing TIGGE multimodel forecasts with reforecastcalibrated ECMWF ensemble forecasts". En: Quarterly Journal of the Royal Meteorological Society 138.668 (oct. de 2012), páginas 1814-1827. ISSN: 00359009. DOI: 10 . 1002/qj . 1895 (citado en página 278).

[11] Hoffman, Ross N y Kalnay, Eugenia. "Lagged average forecasting, an alternative to Monte Carlo forecasting". En: Tellus A 35.2 (1983), páginas 100-118. DoI: 10 . 3402/tellusa . v35i2 . 11425 (citado en páginas 277,282 ).

[12] Hou, Dingchen, KALnAY, Eugenia y DroeGEMEIER, Kelvin K. "Objective verification of the SAMEX'98 ensemble forecasts". En: Monthly Weather Review 129.1 (2001), páginas 73-91 (citado en páginas 278, 284).

[13] Houtekamer, P.L. y Mitchell, Herschel L. "Ensemble Kalman filtering". En: Quarterly Journal of the Royal Meteorological Society 131.613 (oct. de 2005), páginas 3269-3289. ISSN: 00359009. DOI: 10 . 1256/qj . 05.135 (citado en página 279).

[14] Lu, Chungu y col. "Short-Range Numerical Weather Prediction Using Time-Lagged Ensembles". En: Weather and Forecasting 22.3 (jun. de 2007), páginas 580-595. ISSN: 0882- 
8156. DOI: 10.1175/WAF999. 1 (citado en página 277).

[15] Palmer, Tim N. y Hagedorn, Renate. Predictability of weather and climate. Cambridge University Press, 2006 (citado en página 276).

[16] Park, Young-Youn, Buizza, Roberto y Leutbecher, Martin. "TIGGE: Preliminary results on comparing and combining ensembles". En: Quarterly Journal of the Royal Meteorological Society 134.637 (oct. de 2008), páginas 2029-2050. ISSN: 00359009. DOI: 10 . 1002 / qj . 334 (citado en página 278).

[17] Pendergrass, Angeline G y Elmore, Kimberly L. "Ensemble Forecast Bias Correction". En: 4th Annual Student Conference. Amer. Meteor. Soc., San Diego, CA P. 2004, páginas 1-19 (citado en página 277).

[18] Toth, Zoltan y KaLnAY, Eugenia. "Ensemble forecasting at NCEP and the breeding method". En: Monthly Weather Review 125.12 (1997), páginas 3297-3319 (citado en página 279).
[19] Wang, Xuguang y Bishop, Craig H. "A comparison of breeding and ensemble transform Kalman filter ensemble forecast schemes". En: Journal of the atmospheric sciences 60.9 (2003), páginas 1140-1158 (citado en página 279).

[20] Warner, Thomas T., Peterson, Ralph A. y Treadon, Russell E. "A Tutorial on Lateral Boundary Conditions as a Basic and Potentially Serious Limitation to Regional Numerical Weather Prediction". En: Bulletin of the American Meteorological Society 78.11 (nov. de 1997), páginas 2599-2617. ISSN: 0003-0007. DOI: 10 . $1175 / 1520-$ 0477 (1997) 078<2599: ATOLBC>2 . 0 . CO; 2 (citado en página 276).

[21] WEI, Mozheng y col. "Initial perturbations based on the ensemble transform (ET) technique in the NCEP global operational forecast system". En: Tellus A: Dynamic Meteorology and Oceanography 60.1 (ene. de 2008), páginas 62-79. ISSN: 1600-0870. DOI: $10.1111 / j .1600-0870.2007 .00273 . x$ (citado en página 279). 
